\title{
Evidence for a specific cell membrane retinol-binding protein transport mechanism in a human keratinocyte line
}

\author{
JANET HUANG and AMANDIO VIEIRA
}

\author{
Endocrine and Metabolic Research Laboratory, Faculty of Applied Sciences and \\ Institute for Health Research (IHRE), Simon Fraser University, Burnaby, BC, Canada
}

Received September 26, 2005; Accepted November 24, 2005

\begin{abstract}
The epidermis is highly sensitive to retinoids, and vitamin A (retinol) is a critical factor in the regulation of skin cell differentiation and proliferation. Despite extensive knowledge of retinoid-mediated gene transcription effects on epidermal cells and evidence for retinoid-mediated suppression of carcinogenesis in skin, basic transport events, especially cellular uptake, of this vitamin remain poorly understood and controversial. Herein, evidence is presented for receptormediated uptake of retinol-binding protein, RBP, the specific circulatory vitamin A carrier, in the A431 human epidermal cell line. Cellular RBP uptake was significantly inhibited by anti-RBP IgG. Addition of transthyretin (TTR), a circulatory protein that can interact with $\mathrm{RBP}$, to the internalization assay also significantly reduced RBP uptake to $49.4 \pm 4.6 \%$ ( \pm SEM) of control values $(\mathrm{p}<0.01)$. RBP uptake was impaired by sucrose, a known inhibitor of early endocytosis, but not significantly affected by a disruptor of later trafficking events, chlorpromazine. Binding analysis indicated saturable RBP binding to the cell surface and a total of about 94,000 binding sites/cell. Based on dissociation constants, two RBP binding sites were detected with a 50-fold affinity difference: 0.7 and $35.0 \mathrm{nM}$, with 12,000 and 82,000 receptors/cell, respectively. These results indicate that high affinity RBP receptors capable of internalizing RBP independently of TTR exist in these malignant keratinocytes, and that TTR influences binding of RBP to its putative receptor(s). Overall, the data establish membrane transport parameters for RBP, and provide a basis for examining modulation of vitamin A endocytosis that may accompany changes in proliferation or differentiation state of epidermal cells.
\end{abstract}

Correspondence to: Dr Amandio Vieira, Endocrine and Metabolic Research Laboratory, Faculty of Applied Sciences, Simon Fraser University, K9600, 8888 University Drive, Burnaby, BC V5A 1S6, Canada

E-mail: avvieira@sfu.ca

Key words: epidermal cells, transport, vitamin A, retinol-binding protein, transthyretin

\section{Introduction}

Biologically active derivatives of vitamin A (retinol) such as 9-cis and all-trans-retinoic acid are involved in a wide range of physiological regulatory processes. At the molecular and cellular level, this regulation involves control of cell proliferation and differentiation through retinoid-dependent effects on gene expression (reviewed in refs. 1-4). These effects are critical for maintaining skin health and suppressing carcinogenesis in skin $(1,2,4-6)$. Despite extensive knowledge of various retinoid-dependent gene expression effects in epidermal and other cell types, however, much less is known about retinoid transport events at the cell membrane; and endocytosis of circulatory vitamin A carrier proteins by epidermal cells or epidermoid cancer cells remains poorly understood and controversial (see below).

A specific extracellular transport system for retinol exists in humans and other vertebrates and is mediated by the circulatory retinol-binding protein (RBP) (reviewed in refs. 3,7,8). This protein, of about $20 \mathrm{kDa}$, is a member of the lipocalin protein family. A fraction of RBP in the circulation is found associated with a homotetrameric protein, transthyretin (TTR) (reviewed in refs. 3,7,8). TTR is also one of the circulatory carries of thyroid hormones. The retinol-RBP-TTR and retinol-RBP complexes are the specific human circulatory retinol transport complexes (less specific transporters such as albumin and lipoproteins can also carry some forms of vitamin A) (reviewed in refs. 3,7,8).

The possible roles of RBP and TTR in mediating cellular retinol uptake are not well defined. There is controversy regarding cell membrane receptors for RBP or TTR and receptor-independent cellular uptake mechanisms for this vitamin. In several tissues and cell types, cell membrane receptors for RBP or TTR have been reported based on binding studies, and both are candidates for mediating the endocytosis of retinol (reviewed in ref. 3; see Discussion for additional references). A controversy exists regarding cell surface RBP receptors in keratinocytes. There is evidence both for $(9,10)$ and against $(11,12)$ the existence of such receptors.

In the present study, we provide evidence for an RBP receptor in A431 cells, a human cutaneous squamous cell carcinoma cell line. We characterize several basic transport parameters including affinity of the receptor-ligand interaction, number of cell surface binding sites, and sensitivity to inhibition by several endocytic transport modulators. Furthermore, our 
data suggest that these cells can internalize RBP independent of the interaction with TTR.

\section{Materials and methods}

Materials and preparation of biotinylated protein. Transferrin (Tf), transthyretin (TTR), retinol-binding protein (RBP), sucrose, all-trans-retinoic acid (RA), avidin, and horse heart cytochrome c, were obtained from Sigma-Aldrich. Anti-cytochrome c monoclonal antibody was obtained from Spectral Diagnostics, anti-RBP monoclonal IgG from BD Biosciences, and anti-mouse IgG-peroxidase (HRP) conjugate was obtained from Santa Cruz Biotechnology. The malignant keratinocytes, human epidermoid squamous carcinoma cells (A431 cell line) were obtained from ATCC and cultured according to the supplier's recommendations (see also below). The sources of other materials are given below.

The biotinylation reagent, $N$-hydroxy-succinimido-biotin (Sigma) was dissolved in dimethylformamide at $6 \mathrm{mg} / \mathrm{ml}$. For labelling, purified human RBP was dissolved in PBS (phosphate-buffered saline, $\mathrm{pH} 7.4$ ) at $2.5 \mathrm{mg} / \mathrm{ml}$. The biotinylation reaction was performed in PBS by incubation for $1 \mathrm{~h}$ at room temperature at a 10:1 molar ratio of biotinylation reagent to protein; Tris-buffer ( $1 \mathrm{mM}$ final concentration) was subsequently added and the biotin-protein conjugate was aliquoted and stored at $-70^{\circ} \mathrm{C}$.

Cell internalization assay. Human epidermoid squamous carcinoma cells (A431 cell line) were maintained in DMEM (Gibco) supplemented with 10\% FBS (Sigma-Aldrich) in a humidified incubator (VMR), $5 \% \mathrm{CO}_{2}, 37^{\circ} \mathrm{C}$. Cells were grown to a confluency of approximately $80-90 \%$, and then incubated overnight in serum-free medium. They were then gently rinsed in $37^{\circ} \mathrm{C} \mathrm{PBS}$, and incubated $1 \mathrm{~h}$ at $37^{\circ} \mathrm{C}$ in SPB solution $(1: 1$ volume ratio of PBS containing $0.2 \%$ bovine serum albumin: serum-free medium). At various times during this incubation, biochemical reagents were added (given below and in Figure legends) followed by the addition of biotinylated RBP. Endocytosis of biotin-RBP was allowed to occur for a period of $10 \mathrm{~min}$ at $37^{\circ} \mathrm{C}$. After biotin-RBP internalization, cells were washed 3 times with acidic buffer, $25 \mathrm{mM}$ acetic acid and $100 \mathrm{mM} \mathrm{NaCl}, \mathrm{pH} 3.0$, followed by neutralization with SPB, and lysis with ST buffer at $37^{\circ} \mathrm{C}(1 \%$ Triton $\mathrm{X}-100,0.1 \%$ SDS in $1 \mathrm{mM}$ EDTA, $50 \mathrm{mM} \mathrm{NaCl}$ and $10 \mathrm{mM}$ Tris- $\mathrm{HCl}$ ). Internalized biotinylated-protein was measured by ELISA (see below).

In some cases (see also Figures), pretreatment of the cells were performed before addition of biotin-RBP: $400 \mathrm{mM}$ sucrose (Sigma-Aldrich) for $30 \mathrm{~min}$, or $28 \mu \mathrm{M}$ chlorpromazine (Pouleuc) for $1 \mathrm{~h}$, or $1 \mu \mathrm{M}$ all-trans-retinoic acid (SigmaAldrich) for $1 \mathrm{~h}$.

Surface binding assay of biotin-RBP. Attached cells, $1.1 \times 10^{6}$ per $3.5-\mathrm{cm}$ cell culture dish, were incubated overnight with serum-free medium and then gently washed twice in ice-cold PBS and once in ice-cold SPB (see above). Biotinylated protein suspended in ice-cold SPB buffer was added to the cells on ice and incubated for $4 \mathrm{~h}$ at $4^{\circ} \mathrm{C}$. To control for nonspecific binding, a 100 molar excess of non-biotinylated RBP was included in the incubation with the biotin-RBP. Cells

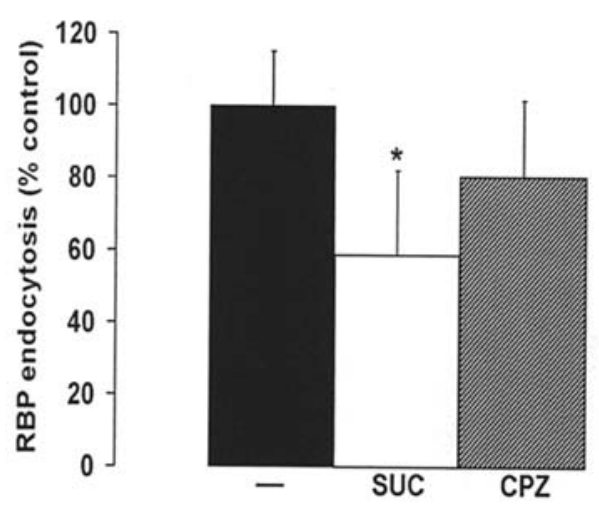

Figure 1. Effects of sucrose and chlorpromazine on RBP endocytosis. Cells were treated with $28 \mu \mathrm{M}$ chlorpromazine (CPZ) or $400 \mathrm{mM}$ sucrose (SUC), or not pretreated (-) before addition of biotin-RBP (see Materials and methods). Internalization of the labeled protein was measured after an incubation for $10 \mathrm{~min}$ at $37^{\circ} \mathrm{C}$. Sucrose, but not chlorpromazine, exhibited a statistically significant inhibition of early RBP internalization, $\mathrm{p}<0.05$, relative to the non-pretreated control ( $\mathrm{n}=9$ for both treatments).

were then washed with ice-cold SPB solution, and lysed with ST blocking buffer as above with the internalization assay. The cell lysate was then analyzed by ELISA.

ELISA. Avidin-coated wells (96-well Nunc-immuno plates) were prepared by addition of $50 \mu \mathrm{g} / \mathrm{ml}$ avidin in $50 \mathrm{mM}$ sodium bicarbonate buffer, $\mathrm{pH} 9.6$ and incubation overnight at $4^{\circ} \mathrm{C}$. Wells were then rinsed with PBS, ST buffer, and PBS again.

Cell lysates from surface binding assays or internalization assays were loaded into the wells and incubated for $2.5 \mathrm{~h}$ at $4^{\circ} \mathrm{C}$. After rinsing with PBS/ST buffer/PBS, primary antibody was added into the wells and incubated for $2.5 \mathrm{~h}$; this was followed by rinsing and addition of secondary antibody-HRP conjugate with another 2.5-h incubation. Wells were washed with PBS, ST buffer, and PBS again. Peroxidase activity was assayed colorimetrically using $0.4 \mathrm{mg} / \mathrm{ml}$ o-phenylenediamine-dihydrochloride (OPG, Sigma-Aldrich) and $0.02 \%$ hydrogen peroxide in $50 \mathrm{mM} \mathrm{Na}_{2} \mathrm{PO}_{4}$ and $30 \mathrm{mM}$ sodium citrate, $\mathrm{pH}$ 5.0. Color reactions were stopped with $4 \mathrm{~N}$ sulfuric acid. Absorbance at 495 (with $\mathrm{A}_{630}$ subtracted) was measured (Statfax-2100 ELISA plate reader).

Statistics. Results are presented as the group mean \pm standard error of the mean (SEM) for each experimental group, unless noted otherwise. The number of measurements, represented by $n$, is given in figure legends. Comparison between means for the main variables and controls (see figures) was performed by two-tailed t-tests. Three p-value levels are indicated for statistical significance: ${ }^{*} \mathrm{p}=0.05-0.02,{ }^{* *} \mathrm{p}=0.01-0.002$, and ${ }^{* * *} \mathrm{p}<0.001$.

\section{Results}

Sucrose, but not chlorpromazine, significantly inhibits biotinRBP uptake by A431 cells. High sucrose concentration (400 mM), a known inhibitor of early, clathrin-mediated endocytosis, is shown in Fig. 1 to strongly inhibit RBP uptake by A431 cells during a 10 -min incubation at $37^{\circ} \mathrm{C}$. 


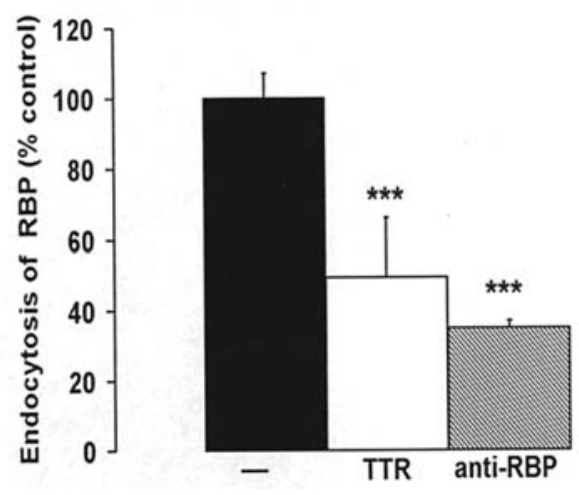

Figure 2. Effects of TTR and anti-RBP IgG on RBP endocytosis. Cells were incubated with biotin-RBP in the absence of any additional added proteins (-), or in the presence of added TTR (TTR), or added anti-RBP IgG (anti$\mathrm{RBP}$ ). The molar ratios for the protein reagents and RBP were $1: 1$ and $1: 1$ for TTR:RBP and IgG:RBP, respectively. Both TTR and anti-RBP IgG exhibited a statistically significant inhibition of early RBP internalization, $\mathrm{p}<0.001$, relative to the control with the added protein competitors $(n=10-14$ for both treatments).

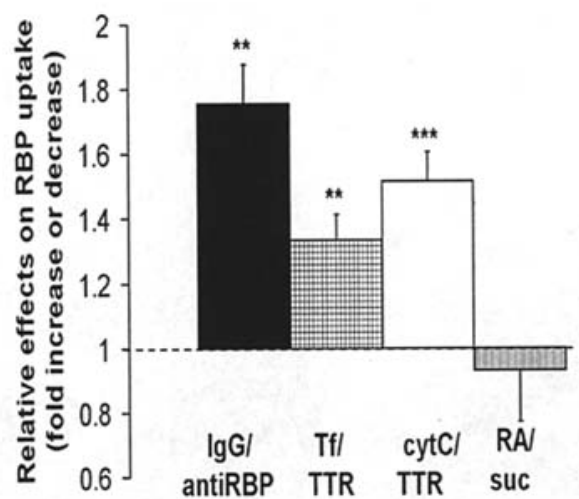

Figure 3. Relative effects of protein and non-protein reagents on RBP endocytosis. Cells were incubated with biotin-RBP in the presence of one of the following: TTR (TTR), anti-RBP IgG (antiRBP), control IgG (IgG), cytochrome c (cyt C), or transferrin (Tf). The respective RBP:protein molar ratios were $1: 1,10: 1,10: 1,1: 1$, and $1: 1$. In addition, two non-protein reagents, sucrose (suc; $400 \mathrm{mM}$ ) and all-trans-retinoic acid (RA; $1 \mu \mathrm{M}$ ) were tested as pretreatments (see Materials and methods and Fig. 1). The relative effects of these reagents on early RBP internalization are shown in the figure as ratios. Thus, aRBP IgG was about 1.7-fold, more potent than control IgG at inhibiting RBP endocytosis; and, likewise, TTR was about 1.3-fold more potent than Tf (a circulatory protein control) and about 1.5-fold more potent than cytochrome c (an intracellular protein control). Although suc significantly inhibits RBP endocytosis (Fig. 1), our results reveal that suc is less potent (about 0.9 times) than RA (see also Results and Discussion).

Chlorpromazine, a known inhibitor of later endocytic events (see Discussion and Fig. 5), does not result in a significant inhibition under these early endocytic assay parameters (Fig. 1). In testing various biochemical reagents for their effects on endocytosis, we noted that retinoic acid (RA) could also potently inhibit RBP uptake by A431 cells; Fig. 3 shows, on a relative scale, that RA $(1 \mu \mathrm{M})$ is slightly more potent at inhibiting RBP uptake than the sucrose treatment.

TTR and anti-RBP IgG both significantly inhibit biotin-RBP uptake by A431 cells. Two RBP-interacting proteins, TTR and an anti-RBP monoclonal antibody, are shown in Fig. 2 to
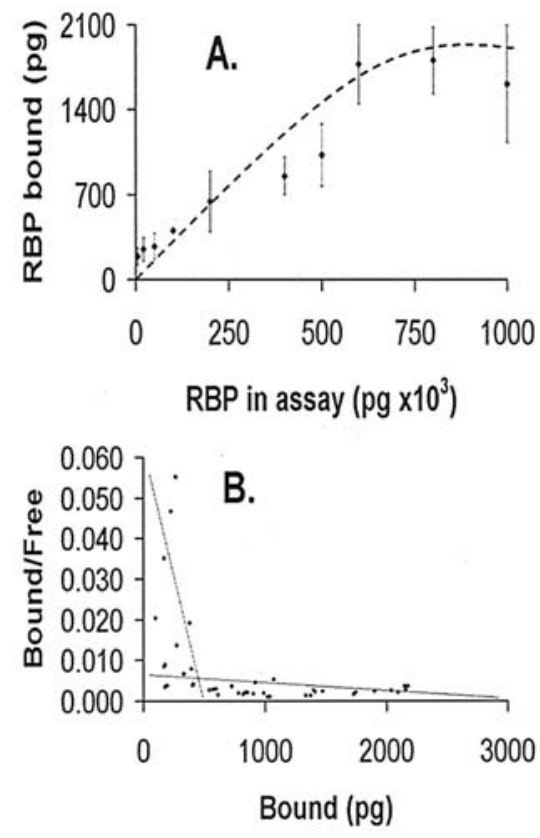

Figure 4. RBP-binding parameters for the human epidermoid squamous carcinoma cells. (A) Specific binding (= total - non-specific bound ligand; see Materials and methods) was performed in the presence of the indicated amounts of biotin-labelled RBP (x axis). (B) Scatchard analysis of specificbinding data suggests the presence of two high-affinity RBP binding sites on the A431 epidermal cells, and a total of about 94,000 binding sites/cell: 82,000 of the lower affinity site $(\mathrm{kd} \sim 1 \mathrm{nM})$ and 12,000 of the higher affinity site $(\mathrm{kd} \sim 35 \mathrm{nM})$. These data represent a pool of different binding assays, $\mathrm{n}=5$.

strongly inhibit RBP uptake by A431 cells at the indicated molar ratios. Fig. 3 provides a summary of relative potencies for the tested RBP endocytosis inhibitors. At a 1:1 molar ratio with RBP, TTR is significantly more potent at inhibiting RBP uptake than two control proteins: transferrin, a circulatory protein, and cytochrome c, an intracellular protein; TTR is about 1.3 and 1.5 times more potent than transferrin or cytochrome c, respectively. Likewise, for the same ratio with $\mathrm{RBP}$, anti-RBP IgG is about 1.7 times more potent than a control IgG (anti-cytochrome c monoclonal antibody) at inhibiting early RBP endocytosis (Fig. 3).

RBP exhibits saturable, high affinity binding to A431 cells with about $9.4 \times 10^{4}$ binding sites/cell. Specific, saturable binding of RBP to A431 cells is shown in Fig. 4A. Upon Scatchard analysis of the binding data (Fig. 4B), the presence of two binding sites with different affinities for RBP is revealed. There are about 12,000 higher affinity sites with $\mathrm{kd}=0.7 \mathrm{nM}$, and 82,000 lower affinity sites with $\mathrm{kd}=35 \mathrm{nM}$, per cell (Fig. 4B).

\section{Discussion}

In this report, the first characterization of RBP transport into A431 epidermoid cells is provided; a putative receptormediated endocytotic process is described (schematic shown in Fig. 5) that occurs preferentially in the absence of TTR, a circulatory protein capable of interacting with RBP or retinoid-RBP complexes. The internalization of circulatory $\mathrm{RBP}$ and, hence, cellular assimilation of circulatory vitamin A 


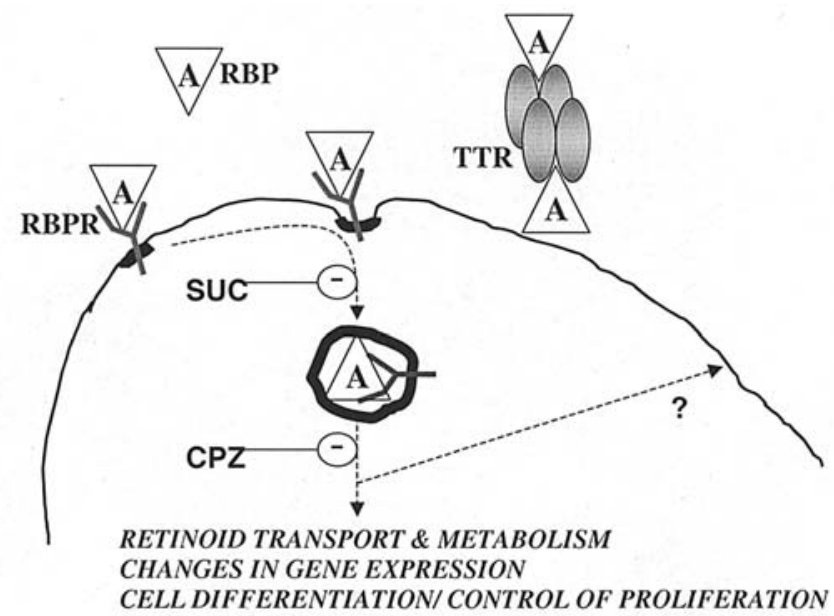

Figure 5. Schematic representation of a possible RBP endocytosis pathway in the A431 cells. RBP (triangles) interacts with a cell surface receptor (RBPR) and is internalized along with its ligand, vitamin A retinol (A), within an endocytic vesicle. Sucrose (SUC) is an inhibitor of this initial internalization by clathrin-coated vesicles. Chlorpromazine (CPZ) inhibits one or more subsequent transport steps. The homotetrameric protein, TTR (shown as four ellipses), may mask the RBP binding site on RBPR and thereby decrease RBP endocytosis (see Discussion for alternative explanation and related references). After unloading the vitamin, the internalized RBP may be (?) recycled as occurs with some other nutrient carrier proteins. Retinol is known to be further transported by cellular binding proteins, and stored or metabolized in the cell. Retinol metabolism can result in the production of bioactive retinoids that influence gene expression and, ultimately, participate in the control of cellular differentiation and proliferation. The effect of retinoids in suppressing carcinogenesis has been reported for many cell types including keratinocytes and other skin cells (see text for references).

is likely to be a critical process in epidermal cell growth and differentiation, and in moderating the risk of epidermal carcinogenesis $(1,2,4-6)$.

The results indicate the presence of two high affinity (kd's of about 1 and $35 \mathrm{nM}$ ) binding sites for RBP with a total of about $9.4 \times 10^{4}$ binding sites per A431 cell. Previous studies with other cell types and membrane preparations (including lipocytes, macrophages, retinal pigment epithelium, hepatocytes, placenta, and visceral yolk sac) have documented both single and double binding sites on cells with an overall $\mathrm{kd}$ range of 2-234 $\mathrm{nM}$ (13-17); the higher affinity sites reported in these studies have dissociation constants under $50 \mathrm{nM}$, a result compatible with our two high affinity sites.

Circulatory RBP, including the holo protein, can exist in free form or complexed with TTR (18-22). Our results indicate that addition of TTR greatly diminishes (by about $50 \%$ ) the amount of RBP endocytosed by the A431 cells (Figs. 2 and 3). A similar reduction in RBP-mediated vitamin A delivery has been observed with macrophages upon addition of TTR (17). TTR likely reduces RBP binding to a putative cell surface receptor either by blocking a receptor binding site on the RBP (shown schematically in Fig. 5) or by inducing a conformational change in RBP structure that lowers its affinity for the receptor $(7,23)$. The transport mechanism that we have characterized is likely sensitive to the different RBP forms that exist in the circulation; and specifically, a free RBP form is proposed to be the primary agent for this suggested vitamin A delivery mechanism. TTR complex formation, thus, could serve in this context to prevent excessive RBPdependent delivery of vitamin A. Retinoids are known to provide a strong growth stimulus for keratinocytes in vivo (reviewed in ref. 4). It is also of interest to note in this regard that a dermotoxic environmental toxin, the dioxin TCDD, can greatly increase the TTR-free, holo-RBP fraction in rat serum (ref. 24 and references therein).

The RBP endocytic process examined in this study was sensitive to sucrose but not chlorpromazine. Sucrose, at the high concentrations used in this study, is a known inhibitor of early clathrin-mediated endocytosis $(25,26)$. Chlorpromazine has been shown to inhibit later endocytic trafficking events such as early-to-late-endosome transport $(26,27)$. Our results indicate that we are indeed measuring early endocytic transport events in our assay $\left(10 \mathrm{~min}, 37^{\circ} \mathrm{C}\right)$, and later events do not significantly contribute to the measured accumulation of RBP in the cells. We report also a strong inhibitory effect of all trans RA on RBP endocytosis. Although further investigation is required to delineate the mechanism for the RA effect, we suggest that one possibility is that RA binds to RBP (28) and affects interaction with the putative receptor; interestingly, in the context of TTR and RA inhibition of RBP endocytosis, RA binding influences a region on the RBP surface that is also involved in TTR binding (29). An alternative possibility for the RA influence on RBP endocytic transport is RAdependent biophysical membrane changes at the cell surface $(30,31)$; in the context of cellular transport, for example, it has been reported that 9-cis RA can affect the process of phagocytosis (32).

In conclusion, a specific transport mechanism for the circulatory vitamin A carrier protein, RBP, has been identified and characterized in the A431 human epidermal cell line. This transport mechanism provides a basis for future experiments to determine if endocytic transport parameters are influenced by factors that affect normal epidermal physiology and pathology. Such factors include vitamin A deficiency and excess, and the proliferation and transformation state of cells.

\section{Acknowledgements}

This study was supported by a grant from Natural Sciences and Engineering Research Council of Canada. We thank P.M. Vieira for help in preparing this manuscript.

\section{References}

1. Clarke N, Germain P, Altucci L and Gronemeyer H: Retinoids: potential in cancer prevention and therapy. Expert Rev Mol Med 6: 1-23, 2004.

2. Verma AK: Retinoids in chemoprevention of cancer. J Biol Regul Homeost Agents 17: 92-97, 2003.

3. Vieira A: Retinoid endocrinology: from metabolism to cellular signalling. In: Subcellular Biochemistry: Fat-Soluble Vitamins. Vol. 30. Quinn PJ and Kagan VE (eds). Academic Press, New York, pp29-51, 1998.

4. Fisher GJ and Voorhees JJ: Molecular mechanisms of retinoid actions in skin. FASEB J 10: 1002-1013, 1996.

5. Chen CF, Goyette P and Lohnes D: RARgamma acts as a tumor suppressor in mouse keratinocytes. Oncogene 23: 5350-5359, 2004.

6. Mrass P, Rendl M, Mildner M, Gruber F, Lengauer B, Ballaun C, Eckhart L and Tschachler E: Retinoic acid increases the expression of p53 and proapoptotic caspases and sensitizes keratinocytes to apoptosis: a possible explanation for tumor preventive action of retinoids. Cancer Res 64: 6542-6548, 2004. 
7. Zanotti G and Berni R: Plasma retinol-binding protein: structure and interactions with retinol, retinoids, and transthyretin. Vitam Horm 69: 271-295, 2004.

8. Quadro L, Hamberger L, Colantuoni V, Gottesman ME and Blaner WS: Understanding the physiological role of retinolbinding protein in vitamin A metabolism using transgenic and knockout mouse models. Mol Aspects Med 24: 421-430, 2003.

9. Torma $\mathrm{H}$ and Vahlquist A: Vitamin A uptake by human skin in vitro. Arch Dermatol Res 276: 390-395, 1984.

10. Vahlquist A and Torma H: Retinol uptake by human keratinocytes - receptor-mediated or not? J Invest Dermatol 99: 512-514, 1992.

11. Creek KE, Silverman-Jones CS and De Luca LM: Comparison of the uptake and metabolism of retinol delivered to primary mouse keratinocytes either free or bound to rat serum retinolbinding protein. J Invest Dermatol 92: 283-289, 1989.

12. Hodam JR, St Hilaire P and Creek KE: Comparison of the rate of uptake and biologic effects of retinol added to human keratinocytes either directly to the culture medium or bound to serum retinol-binding protein. J Invest Dermatol 97: 298-304, 1991.

13. Fortuna VA, Martucci RB, Trugo LC and Borojevic R: Hepatic stellate cells uptake of retinol associated with retinol-binding protein or with bovine serum albumin. J Cell Biochem 90: 792-805, 2003.

14. Mansouri A, Gueant JL, Capiaumont J, Pelosi P, Nabet P and Haertle T: Plasma membrane receptor for beta-lactoglobulin and retinol-binding protein in murine hybridomas. Biofactors 7: 287-298, 1998.

15. Sivaprasadarao A and Findlay JB: The interaction of retinolbinding protein with its plasma-membrane receptor. Biochem $\mathrm{J}$ 255: 561-569, 1988.

16. Senoo H, Smeland S, Malaba L, Bjerknes T, Stang E, Roos N, Berg T, Norum KR and Blomhoff R: Transfer of retinol-binding protein from HepG2 human hepatoma cells to cocultured rat stellate cells. Proc Nat Acad Sci USA 90: 3616-3620, 1993.

17. Hagen E, Myhre AM, Smeland S, Halvorsen B, Norum KR and Blomhoff R: Uptake of vitamin A in macrophages from physiologic transport proteins: role of retinol-binding protein and chylomicron remnants. J Nutr Biochem 10: 345-352, 1999.

18. Jaconi S, Saurat JH and Siegenthaler G: Analysis of normal and truncated holo- and apo-retinol-binding protein (RBP) in human serum: altered ratios in chronic renal failure. Eur J Endocrinol 134: 576-582, 1996.

19. Burri BJ, Neidlinger TR and Zwick H: Comparison of the properties and concentrations of the isoforms of retinolbinding protein in animals and human beings. Am J Vet Res 54: $1213-1220,1993$.
20. Burri BJ, Kutnink MA and Neidlinger TR: Assay of human transthyretin-bound holo-retinol-binding protein with reversephase high-performance liquid chromatography. Chromatography 567: 369-380, 1991.

21. Jaconi S, Rose K, Hughes GJ, Saurat JH and Siegenthaler G: Characterization of two post-translationally processed forms of human serum retinol-binding protein: altered ratios in chronic renal failure. J Lipid Res 36: 1247-1253, 1995.

22. Nedelkov D and Nelson RW: Delineating protein-protein interactions via biomolecular interaction analysis-mass spectrometry. J Mol Recognit 16: 9-14, 2003.

23. Naylor HM and Newcomer ME: The structure of human retinolbinding protein (RBP) with its carrier protein transthyretin reveals an interaction with the carboxy terminus of RBP. Biochemistry 38: 2647-2653, 1999.

24. Brouwer A, Hakansson H, Kukler A, van den Berg KJ and Ahlborg UG: Marked alterations in retinoid homeostasis of Sprague-Dawley rats induced by a single i.p. dose of $10 \mathrm{mg} / \mathrm{kg}$ of 2,3,7,8-tetrachlorodibenzo-p-dioxin. Toxicology 58: 267-283, 1989.

25. Heuser JE and Anderson RG: Hypertonic media inhibit receptormediated endocytosis by blocking clathrin-coated pit formation. J Cell Biol 108: 389-400, 1989.

26. Shogomori $\mathrm{H}$ and Futerman $\mathrm{AH}$ : Cholera toxin is found in detergent-insoluble rafts/domains at the cell surface of hippocampal neurons but is internalized via a raft-independent mechanism. J Biol Chem 276: 9182-9188, 2001.

27. Wang LH, Rothberg KG and Anderson RG: Mis-assembly of clathrin lattices on endosomes reveals a regulatory switch for coated pit formation. J Cell Biol 123: 1107-1117, 1993.

28. Malpeli G, Folli C and Berni R: Retinoid binding to retinolbinding protein and the interference with the interaction with transthyretin. Biochim Biophys Acta 1294: 48-54, 1996.

29. Zanotti G, Marcello M, Malpeli G, Folli C, Sartori G and Berni R: Crystallographic studies on complexes between retinoids and plasma retinol-binding protein. J Biol Chem 269: 29613-29620, 1994.

30. Tzankov A: Retinoic acid-induced Golgi apparatus disruption in F2000 fibroblasts: a model for enhanced intracellular retrograde transport. J Biochem Mol Biol 36: 265-268, 2003.

31. Wu YN, Gadina M, Tao-Cheng JH and Youle RJ: Retinoic acid disrupts the Golgi apparatus and increases the cytosolic routing of specific protein toxins. J Cell Biol 125: 743-753, 1994.

32. Serghides L and Kain KC: Mechanism of protection induced by vitamin A in falciparum malaria. Lancet 359: 1404-1406, 2002 . 